\title{
Simulation of multi-band MIMO FMCW radar performance in detecting maritime vessels
}

\begin{abstract}
The objective of this paper is to investigate the range detection performance of co-located multi-band multiple-input multiple output (MIMO) Frequency Modulated Continuous Wave (FMCW) radar using various MIMO configurations, in detecting a slow radar cross sections (RCS) fluctuating vessel via a numerical simulation. The simulated MIMO FMCW radar is emitting multiple triangular FMCW waveforms with each baseband sweeps at different frequency range. At the MIMO radar receiver's ends, a proposed spectrum averaging scheme is applied to combine the receiving signals before implementing conventional ranging algorithms. A $3 \mathrm{MHz}$ interval band is introduced between sub-bands and its effect had been observed. Performance of the proposed system was evaluated in terms of probability of range error against signal-to-noise ratio (SNR). From the simulation, it was observed that $3 \times 3$ MIMO performed 1 to $2 \mathrm{~dB}$ better compared to other topologies at $20 \%$ probability of range error.
\end{abstract}

Keyword: FMCW; Radar; Swerling; SISO; MIMO; Range; Radar detection 